\title{
SERUM CYTOKINES AND EEG ABNORMALITIES WITH INFLUENZA ASSOCIATED FEBRILE SEIZURES
}

The relation between serum cytokine levels, EEG changes, and mild neurological complications (delirium and febrile seizure) in 27 children with proven influenza infection was studied at Okazaki City Hospital, and other centers in Japan. Ten patients had delirium (4 also had a febrile seizure) and 17 had febrile seizures. Febrile delirium was characterized by meaningless speech, periodic inappropriate crying, blank stare, and hallucinations. EEGs recorded in 11 cases within 10-33 hours after a seizure showed focal or generalized slowing in 5 . EEGs in 5 patients recorded 36 hours or more after onset of the neurologic complication were normal in 3 . The median serum level of interleukin-6 (IL-6) was $31.2+/-15.1 \mathrm{pg} / \mathrm{ml}$ in the delirium group, $42.3+/-44.0 \mathrm{pg} / \mathrm{ml}$ in the febrile seizure group, and $15.4+/-7.0 \mathrm{pg} / \mathrm{ml} \mathrm{in}$ the control group. Serum IL-6 level differed significantly between the delirium/ febrile seizure groups and control group (each p<0.05). IL-6 reached $196 \mathrm{pg} / \mathrm{ml}$ in one child with a generalized seizure lasting $5 \mathrm{~min}$. IL-6 levels were highest in infants with generalized EEG slowing and lowest in those with a normal EEG.

Tumor necrosis factor-x (TNF-x) was less than $5 \mathrm{pg} / \mathrm{ml}$ in most patients with delirium, febrile seizures, and in the control group. TNF-x and soluble tumor necrosis factor receptor-1 (sTNFR-1) levels were not significantly different from controls. In 2 patients with influenza encephalopathy manifested by clustered or prolonged seizures, impaired consciousness, EEG slowing, and fatal brain edema in one, IL-6 levels were markedly elevated (117 and $262 \mathrm{pg} / \mathrm{ml}$ ), whereas sTNFR-1 was not increased (TNF-x was not measured).

Serum IL-6 levels and EEG changes may prove useful indicators for the early diagnosis and differentiation of mild (delirium and febrile seizures) and severe (encephalopathy) neurologic complications of influenza infection. (Fukumoto Y, Okumura A, Hayakawa Fet al. Serum levels of cytokines and EEG findings in children with influenza associated with mild neurological complications. Brain Dev July 2007;29:425-430). (Respond: Dr Yukiko Fukumoto, Department of Pediatrics, Okazaki City Hospital, Okasaki, Japan. E-mail: fqmtykk@yahoo.co.jp).

COMMENT. Proinflammatory cytokines are increased in influenza-associated febrile seizures and encephalopathy, and serum levels of IL-6 and IL-10 are useful indicators of the severity of the disease (Kawada J-I et al. J Infect Dis 2003;188:690-698). Some viruses particularly prone to cause encephalopathy or complex febrile seizures result in excessive systemic immune and cytokine responses ("Role of viral infections in the etiology of febrile seizures" reviewed in Pediatr Neurol 2006;35:165-172). Notwithstanding some negative findings, most studies demonstrate an elevated IL-6, favoring a cytokine role in febrile seizures, in addition to the association with encephalopathy. An observed increase in CSF cytokine levels attributed to an increased permeability of the blood-CSF barrier has been associated with severe encephalopathy and a poor prognosis. 